\title{
ZEROS OF AN ALGEBRAIC POLYNOMIAL WITH NONEQUAL MEANS RANDOM COEFFICIENTS
}

\author{
K. FARAHMAND and P. FLOOD
}

Received 16 July 2004

\begin{abstract}
This paper provides an asymptotic estimate for the expected number of real zeros of a random algebraic polynomial $a_{0}+a_{1} x+a_{2} x^{2}+\cdots+a_{n-1} x^{n-1}$. The coefficients $a_{j}(j=$ $0,1,2, \ldots, n-1)$ are assumed to be independent normal random variables with nonidentical means. Previous results are mainly for identically distributed coefficients. Our result remains valid when the means of the coefficients are divided into many groups of equal sizes. We show that the behaviour of the random polynomial is dictated by the mean of the first group of the coefficients in the interval $(-1,1)$ and the mean of the last group in $(-\infty,-1) \cup(1, \infty)$.

2000 Mathematics Subject Classification: 60H99, 42Bxx.
\end{abstract}

1. Introduction. There are many known asymptotic estimates for the expected number of real zeros of polynomials

$$
P_{n}(x)=\sum_{j=0}^{n-1} a_{j} x^{j},
$$

with random coefficients $a_{0}, a_{1}, \ldots, a_{n-1}$. These estimates, however, are mainly devoted for the case of identically distributed coefficients, albeit for different classes of distributions. From the pioneer work of Kac [9], it is known that in the normal standard case the expected number of real zeros of $P_{n}(x)$, denoted by $\mathrm{EN}_{n}(-\infty, \infty)$, is asymptotic to $(2 / \pi) \log n$ as $n \rightarrow \infty$. Later, the error term involved in this asymptotic formula is significantly reduced by Wilkins [12], using a new approach. Also, various cases of dependent coefficients are studied mainly by Sambandham, see, for example, [1, 11].

For a case when the coefficients have a nonzero mean, $\mu$, it is shown that $\mathrm{EN}_{n}(-\infty, \infty)$ is reduced by half to $(1 / \pi) \log n$. In [4], a case when the coefficients are nonidentical is considered. However, they are only permitted to belong to two groups of distributions. In an interesting work, Edelman and Kostlan [3] consider a case of nonidentically distributed coefficients, each having different variances. It is important to note that so far their coefficients are the only type of distribution which increases the order of numbers of real zeros from $O(\log n)$ to $O(\sqrt{n})$. In [6], all the coefficients of (1.1) have also different means, but they follow a geometric progression, that is, $E\left(a_{j}\right)=\mu^{j}$, $j=0,1,2, \ldots, n-1$ for a constant $\mu$. Recent developments on the subject as well as related works are reviewed in [5].

Here, we assume that the coefficients $\left\{a_{j}\right\}_{j=0}^{n-1}$ in (1.1) are independent and nonidentically distributed. They are normal random variables, all have variance one and their 
means are divided into groups of equal size. Let $E\left(a_{j}\right)=\mu_{1}$ for $0 \leq j \leq n_{1}-1, E\left(a_{j}\right)=\mu_{2}$ for $n_{1} \leq j \leq n_{2}-1, \ldots, E\left(a_{j}\right)=\mu_{k}$ for $n_{k-1} \leq j \leq n-1$, where $n_{1}=[n / k], n_{2}=[2 n / k]$, and so forth and $k$ is any constant such that $1<k \leq \gamma / 5, \gamma$ a constant to be defined below. In this work, we impose the conditions that $\mu_{1}$ and $\mu_{k}$ are bounded away from zero, that is, $\left|\mu_{1}\right| \sqrt{\log n} \rightarrow \infty$ and $\left|\mu_{k}\right| \sqrt{\log n} \rightarrow \infty$ as $n \rightarrow \infty$. Also $\left|\mu_{j}-\mu_{j-1}\right| \leq c$, where $c=O\left(n^{r-5} \log n\right)=\mu_{1}^{2}$ and $r$ is defined below. Let $N_{n}(\alpha, \beta)$ represent the number of real zero crossings of $P_{n}(x)$ on the interval $(\alpha, \beta)$. In these circumstances, we have the following theorem.

THEOREM 1.1. If the coefficients of $P_{n}(x)$ in (1.1) are as outlined above, then the mathematical expectation of the number of zeros of $P_{n}(x)$ on various intervals of the real number line, denoted as $E N_{n}(\alpha, \beta)$, satisfies

$$
\mathrm{EN}_{n}(0,1)=O(\sqrt{\log n}), \quad \mathrm{EN}_{n}(-1,0) \sim\left(\frac{1}{2 \pi}\right) \log n
$$

if $\left|\mu_{1}\right|(\log n)^{1 / 2} \rightarrow \infty$ as $n \rightarrow \infty$ and $\mu_{2}, \ldots, \mu_{k}=o\left(n^{q}\right) ;$ and

$$
\mathrm{EN}_{n}(-\infty,-1) \sim\left(\frac{1}{2 \pi}\right) \log n, \quad \mathrm{EN}_{n}(1, \infty)=O(\sqrt{\log n})
$$

if $\left|\mu_{k}\right|(\log n)^{1 / 2} \rightarrow \infty$ as $n \rightarrow \infty$ and $\mu_{1}, \ldots, \mu_{k-1}=o\left(n^{q}\right)$, where $q$ is any positive constant.

2. Proof of the theorem. We start our proof by noting that in the interval $(0,1)$ $x^{n}<x^{n_{k-1}}<\cdots<x^{n_{2}}<x^{n_{1}} \leq \exp \left(-n_{1} \varepsilon\right)=n^{-r}$, where $r=\gamma / k$ and $\varepsilon=n^{-a}, a=$ $1-\log \log n^{\gamma} / \log n$. First, for $0<x<1-\varepsilon$ we define and expand the required variances and covariances as follows:

$$
\begin{aligned}
A^{2} & =\operatorname{var}\left\{P_{n}(x)\right\}=\sum_{j=0}^{n-1} x^{2 j}=\frac{\left(1-x^{2 n}\right)}{1-x^{2}}=\frac{1}{1-x^{2}}+O\left\{n^{1-2 r}(\gamma \log n)^{-1}\right\} \\
B^{2} & =\operatorname{var}\left\{P_{n}^{\prime}(x)\right\}=\sum_{j=0}^{n-1} j^{2} x^{2 j-2}=\frac{\left(1+x^{2}\right)\left(1-x^{2 n}\right)}{\left(1-x^{2}\right)^{3}}-\frac{2 n x^{2 n}}{\left(1-x^{2}\right)^{2}}-\frac{n^{2} x^{2 n-2}}{1-x^{2}} \\
& =\frac{\left(1+x^{2}\right)}{\left(1-x^{2}\right)^{3}}+O\left\{n^{5-2 r}(\gamma \log n)^{-3}\right\}, \\
C & =\operatorname{cov}\left\{P_{n}(x), P_{n}^{\prime}(x)\right\}=\sum_{j=0}^{n-1} j x^{2 j-1}=\frac{x\left(1-x^{2 n}\right)}{\left(1-x^{2}\right)^{2}}-\frac{n x^{2 n-1}}{1-x^{2}} \\
& =\frac{x}{\left(1-x^{2}\right)^{2}}+O\left\{n^{3-2 r}(\gamma \log n)^{-2}\right\} .
\end{aligned}
$$

The expected values that give this problem its special properties are defined as

$$
m=E\left\{P_{n}(x)\right\}, \quad m^{\prime}=E\left\{P_{n}^{\prime}(x)\right\} .
$$


These definitions can easily be expanded to show that

$$
\begin{aligned}
& m=\mu_{1} \sum_{j=0}^{n_{1}-1} x^{j}+\mu_{2} \sum_{j=n_{1}}^{n_{2}-1} x^{j}+\cdots+\mu_{k} \sum_{j=n_{k-1}}^{n-1} x^{j}, \\
& m^{\prime}=\mu_{1} \sum_{j=0}^{n_{1}-1} j x^{j-1}+\mu_{2} \sum_{j=n_{1}}^{n_{2}-1} j x^{j-1}+\cdots+\mu_{k} \sum_{j=n_{k-1}}^{n-1} j x^{j-1} .
\end{aligned}
$$

These in turn give

$$
\begin{aligned}
& \left|m-\frac{\mu_{1}\left(1-x^{n}\right)}{1-x}\right| \leq \frac{c x^{n_{1}}}{(1-x)\left(1-x^{n_{1}}\right)}, \\
& \left|m^{\prime}-\left[\frac{\mu_{1}\left(1-x^{n}\right)}{(1-x)^{2}}-\frac{\mu_{1} x^{2 n_{1}}}{x(1-x)}\right]\right| \\
& \leq c x^{n_{1}}\left\{1-\left(x^{n_{1}}\right)^{k_{1}-1}-\left(1-x^{n_{1}}\right)\left(k_{1}-1\right)\left(x^{n_{1}}\right)^{k_{1}-1}\right. \\
& \quad+(1-x) \frac{\left(x^{n_{1}}\left[1-\left(x^{n_{1}}\right)^{k_{1}-1}\right]-\left(1-x^{n_{1}}\right)\left(k_{1}-1\right) x^{n}\right)}{x\left(1-x^{n_{1}}\right)^{2}} \\
& \left.\quad+\frac{(1-x)\left[n_{1}-\left(k_{1}-1\right) n_{1}\left(x^{n_{1}}\right)^{k_{1}-2}\right]}{x}\right\} \times \frac{1}{(1-x)^{2}\left(1-x^{n_{1}}\right)} .
\end{aligned}
$$

To highlight the dominant terms on $0<x<1-\varepsilon$, we can write $m$ and $m^{\prime}$ as

$$
\begin{aligned}
m & =\frac{\mu_{1}}{1-x}+O\left\{\left(\left|\mu_{1}\right|+c\right) n^{1-r} \gamma \log n\right\}, \\
m^{\prime} & =\frac{\mu_{1}}{(1-x)^{2}}+O\left\{\left(\left|\mu_{1}\right|+c\right) n^{2-r} \gamma \log n\right\} .
\end{aligned}
$$

Kac [9] obtained a formula for the average number of real zeros of a polynomial of the form (1.1), where the coefficients are independent standard normal random variables. Hammersley [8] in his work on the zeros of polynomials with generally distributed coefficients, and Cramér and Leadbetter [2] in their study of nonstationary random processes, developed the findings of Kac [9] and Rice [10] for more general situations. From these, Farahmand and Hannigan [7] obtained an expression of the Kac-Rice formula for independent identically distributed normal coefficients having nonzero mean. Although in this case we study a polynomial that has nonidentically distributed coefficients, we can utilise the same Kac-Rice formula. Hence, from [7],

$$
\begin{aligned}
\operatorname{EN}_{n}(\alpha, \beta)= & \int_{\alpha}^{\beta}\left(\frac{\Delta}{\pi A^{2}}\right) \exp \left\{\frac{-\left(A^{2} m^{\prime 2}+B^{2} m^{2}-2 C m m^{\prime}\right)}{2 \Delta^{2}}\right\} d x \\
& +\int_{\alpha}^{\beta}\left(\frac{\sqrt{2}}{\pi}\right)\left(A^{2} m^{\prime}-C m\right) A^{-3} \exp -\left(\frac{m^{2}}{2 A^{2}}\right) \times \operatorname{erf}\left(\frac{\left[A^{2} m^{\prime}-C m\right]}{A \Delta \sqrt{2}}\right) d x \\
= & I_{1}(\alpha, \beta)+I_{2}(\alpha, \beta),
\end{aligned}
$$


where $\Delta^{2}=A^{2} B^{2}-C^{2}$. By (2.1) to (2.8), we can establish that

$$
\begin{gathered}
\frac{\Delta}{A^{2}}=\frac{1}{1-x^{2}}+O\left\{n^{4-2 r}(\gamma \log n)^{-4}\right\} \\
\frac{\left(A^{2} m^{\prime 2}+B^{2} m^{2}-2 C m m^{\prime}\right)}{2 \Delta^{2}}=\frac{\mu_{1}^{2}(1+x)}{1-x}+O\left\{\frac{\mu_{1}\left(\left|\mu_{1}\right|+c\right)}{n^{r-1}}\right\} .
\end{gathered}
$$

These, in turn, give the value of $I_{1}(0,1-\varepsilon)$ in (2.9) as

$$
\begin{aligned}
I_{1}(0,1-\varepsilon)= & \int_{0}^{1-\varepsilon}\left(\frac{\Delta}{\pi A^{2}}\right) \exp \left\{-\frac{\left(A^{2} m^{\prime 2}+B^{2} m^{2}-2 C m m^{\prime}\right)}{2 \Delta^{2}}\right\} d x \\
= & \pi^{-1} \int_{0}^{1-\varepsilon}\left(1-x^{2}\right)^{-1}\left[\exp \left\{-\frac{\mu_{1}^{2}(1+x)}{1-x}\right\}+O\left\{\frac{\mu_{1}\left(\left|\mu_{1}\right|+c\right)}{n^{r-1}}\right\}\right] d x \\
& \times\left\{1+O\left\{n^{4-2 r}(\gamma \log n)^{-4}\right\}\right\} \\
\leq & \pi^{-1} \int_{0}^{1-\varepsilon}\left(1-x^{2}\right)^{-1} \exp \left\{-\frac{\mu_{1}^{2}}{1-x}\right\} d x .
\end{aligned}
$$

Putting $t=1 /(1-x)$ gives

$$
I_{1}(0,1-\varepsilon) \leq \pi^{-1} \int_{1}^{1 / \varepsilon} \exp \left(-\mu_{1}^{2} t\right) d t=\frac{\left[\exp \left(-\mu_{1}^{2}\right)-\exp \left(-\mu_{1}^{2} / \varepsilon\right)\right]}{\pi \mu_{1}^{2}} \leq \frac{\exp \left(-\mu_{1}^{2}\right)}{\pi \mu_{1}^{2}}
$$

Therefore, for $\mu_{1} \neq 0$ and bounded such that $\left|\mu_{1}\right|(\log n)^{1 / 2} \rightarrow \infty$ as $n \rightarrow \infty$, we have

$$
I_{1}(0,1-\varepsilon)=O(\sqrt{\log n})
$$

In the following, we show that for all values of $\mu$ under consideration, the expected number of real zeros in the interval $(1-\varepsilon, 1)$ is negligible for large $n$. Since, from (2.1)(2.3),

$$
\Delta^{2}=\left(1-x^{2 n}\right)^{2}\left(1-x^{2}\right)^{-4}\left\{1-\frac{n^{2} x^{2 n-2}\left(1-x^{2}\right)^{2}}{\left(1-x^{2 n}\right)^{2}}\right\}
$$

we have

$$
\frac{\Delta}{A^{2}}=\left\{1-\frac{n^{2} x^{2 n-2}\left(1-x^{2}\right)^{2}}{\left(1-x^{2 n}\right)^{2}}\right\}^{1 / 2} \times \frac{1}{1-x^{2}}
$$

This enables us to establish the inequality

$$
\frac{\Delta}{A^{2}}<\sqrt{\frac{(2 n-1)}{1-x}}
$$


on the interval $(1-\varepsilon, 1)$ for all sufficiently large $n$. Therefore, we find

$$
I_{1}(1-\varepsilon, 1)<\int_{1-\varepsilon}^{1}\left(\frac{\Delta}{\pi A^{2}}\right) d x=o(\sqrt{\log n}) .
$$

To evaluate $I_{2}(0,1)$ we note that since $(d / d x)(m / A)=A^{-3}\left(A^{2} m^{\prime}-C m\right)$ and $\operatorname{erf}(x) \leq$ $\sqrt{\pi} / 2$, we have

$$
I_{2}(0,1) \leq\left(\frac{1}{\sqrt{2 \pi}}\right) \int_{\mu_{1}}^{\zeta} \exp \left(-\frac{u^{2}}{2}\right) d u=O(1),
$$

where $u=m / A$ and $\zeta=(\sqrt{n} / k) \sum_{j=1}^{k} \mu_{j}$. Thus, by (2.13), (2.17), and (2.18), we have

$$
\mathrm{EN}_{n}(0,1)=O(\sqrt{\log n})
$$

Given that $P_{n}(x)=x^{n-1} \sum_{j=0}^{n-1} a_{n-j-1} x^{-j}=x^{n-1} P_{n}^{*}(y), y=1 / x$, we can state that for each zero of $P_{n}(x)$ in $(0,1)$ [or $(-1,0)$ ] there is a corresponding zero of $P_{n}^{*}(x)$ in $(1, \infty)$ [or $(-\infty,-1)]$. We interchange $\mu_{1}$ and $\mu_{k}, \mu_{2}$, and $\mu_{k-1}$, and so forth, and replace $k$ with $k /(k-1)$ in $P_{n}^{*}(x)$. The distribution of $P_{n}^{*}(x)$ is therefore identical to that of $P_{n}(x)$. Let $N_{n}^{*}(\alpha, \beta)$ denote the number of zeros of $P_{n}^{*}(x)$ in the interval $(\alpha, \beta)$. Therefore, we have $\operatorname{EN}_{n}(0,1)=\mathrm{EN}_{n}^{*}(1, \infty)$, and $\mathrm{EN}_{n}(-1,0)=\mathrm{EN}_{n}^{*}(-\infty,-1)$.

To find $\mathrm{EN}_{n}(-1,0)$, we let $y=-x$. This gives

$$
\begin{aligned}
I_{1}(-1 & +\varepsilon, 0) \\
= & \pi^{-1} \int_{0}^{1-\varepsilon}\left(1-y^{2}\right)^{-1}\left[\exp \left\{-\frac{\mu_{1}^{2}(1-y)}{1+y}\right\}+O\left\{\frac{\mu_{1}\left(\left|\mu_{1}\right|+c\right)}{n^{r-1}}\right\}\right] d y \\
& \times\left\{1+O\left\{n^{4-2 r}(\gamma \log n)^{-4}\right\}\right\} .
\end{aligned}
$$

We can say that

$$
I_{1}(-1+\varepsilon, 0) \leq \pi^{-1} \int_{0}^{1-\varepsilon}\left(1-y^{2}\right)^{-1} d y \leq\left(\frac{1}{2 \pi}\right) \log n .
$$

It is also true to say that

$$
I_{1}(-1+\varepsilon, 0)>\pi^{-1} \int_{0}^{1-\varepsilon}[2(1-y)]^{-1} \exp \left\{2 \mu_{1}^{2}(1-y)\right\} d y .
$$

Putting $t=2 \mu_{1}^{2}(1-y)$ gives

$$
\begin{aligned}
I_{1}(-1+\varepsilon, 0) & >\left(\frac{1}{2 \pi}\right) \int_{2 \mu_{1}^{2}}^{2 \mu_{1}^{2} \varepsilon}-\left(\frac{1}{t}\right) \exp (-t) d t \\
& =\left(\frac{1}{2 \pi}\right) \int_{2 \mu_{1}^{2} \varepsilon}^{2 \mu_{1}^{2}} \frac{e^{-t}}{t} d t \\
& =\left(\frac{1}{2 \pi}\right) \int_{2 \mu_{1}^{2} \varepsilon}^{2 \mu_{1}^{2}} \frac{d t}{t}-\left(\frac{1}{2 \pi}\right) \int_{2 \mu_{1}^{2} \varepsilon}^{2 \mu_{1}^{2}} \frac{\left(1-e^{-t}\right)}{t} d t .
\end{aligned}
$$


Upon integration, we obtain

$$
I_{1}(-1+\varepsilon, 0)>\left(\frac{1}{2 \pi}\right) \log n+O(\log \log n) .
$$

Thus, by (2.21) and (2.24), we find

$$
I_{1}(-1+\varepsilon, 0) \sim\left(\frac{1}{2 \pi}\right) \log n
$$

By letting $y=-x$, we can establish, in a manner similar to (2.17), that

$$
I_{1}(-1,-1+\varepsilon)=o(\sqrt{\log n})
$$

As regards $I_{2}(-1,0)$, we find that

$$
I_{2}(-1,0) \leq\left(\frac{1}{\sqrt{2 \pi}}\right) \int_{0}^{\mu_{1}} \exp \left(-\frac{\mu^{2}}{2}\right) d u
$$

when $n_{1}$ is even, or for $\tau=\sum_{j=1}^{k}(-1)^{j+1} \mu_{j}$,

$$
I_{2}(-1,0) \leq\left(\frac{1}{\sqrt{2 \pi}}\right) \int_{\tau / \sqrt{n}}^{\mu_{1}} \exp \left(-\frac{u^{2}}{2}\right) d u
$$

when $n_{1}$ is odd. Thus,

$$
I_{2}(-1,0) \leq \frac{1}{2}
$$

We can conclude from (2.25), (2.26), and (2.29) that

$$
\mathrm{EN}_{n}(-1,0) \sim\left(\frac{1}{2 \pi}\right) \log n
$$

This completes the proof of the theorem. The result of this paper is similar to the findings of [4] and establishes the predominant influence of the means of the first and last groups of random coefficients on the average number of zeros of the polynomial. An area of future interest regarding random algebraic polynomials would be to ascertain how small the first and last groups of coefficients can be before the means of other groups become significant. 


\section{REFERENCES}

[1] A. T. Bharucha-Reid and M. Sambandham, Random Polynomials, Probability and Mathematical Statistics, Academic Press, Florida, 1986.

[2] H. Cramér and M. R. Leadbetter, Stationary and Related Stochastic Processes. Sample Function Properties and Their Applications, John Wiley \& Sons, New York, 1967.

[3] A. Edelman and E. Kostlan, How many zeros of a random polynomial are real? Bull. Amer. Math. Soc. (N.S.) 32 (1995), no. 1, 1-37.

[4] K. Farahmand, Real zeros of random algebraic polynomials, Proc. Amer. Math. Soc. 113 (1991), no. 4, 1077-1084.

[5]__ Topics in Random Polynomials, Pitman Research Notes in Mathematics Series, vol. 393, Longman, Harlow, 1998.

[6] K. Farahmand, P. Flood, and P. Hannigan, Zeros of a random algebraic polynomial with coefficient means in geometric progression, J. Math. Anal. Appl. 269 (2002), no. 1, 137-148.

[7] K. Farahmand and P. Hannigan, On the expected number of zero crossings of a random algebraic polynomial, Nonlinear Anal. 30 (1997), no. 2, 1219-1224, Proceedings of the Second World Congress of Nonlinear Analysts, Part 2 (Athens, 1996).

[8] J. M. Hammersley, The zeros of a random polynomial, Proceedings of the Third Berkeley Symposium on Mathematical Statistics and Probability, 1954/1955, Vol. II, University of California Press, California, 1956, pp. 89-111.

[9] M. Kac, On the average number of real roots of a random algebraic equation, Bull. Amer. Math. Soc. 49 (1943), 314-320.

[10] S. O. Rice, Mathematical analysis of random noise, Bell System Tech. J. 24 (1945), 46-156, reprinted in: Selected Papers on Noise And Stochastic Processes (N. Wax, ed.), Dover, New York, 1954, pp. 133-294.

[11] M. Sambandham, On the average number of real zeros of a class of random algebraic curves, Pacific J. Math. 81 (1979), no. 1, 207-215.

[12] J. E. Wilkins Jr., An asymptotic expansion for the expected number of real zeros of a random polynomial, Proc. Amer. Math. Soc. 103 (1988), no. 4, 1249-1258.

K. Farahmand: Department of Mathematics, University of Ulster, Jordanstown, County Antrim BT37 0QB, UK

E-mail address: k. farahmand@u1ster.ac.uk

P. Flood: Department of Mathematics, University of Ulster, Jordanstown, County Antrim BT37 0QB, UK

E-mail address: floodpaddy@eircom. net 


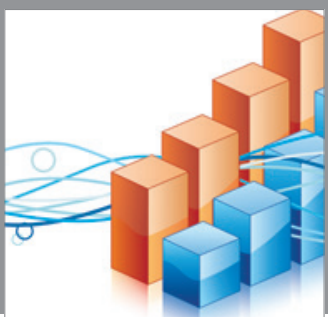

Advances in

Operations Research

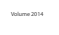

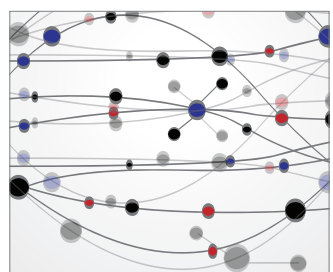

\section{The Scientific} World Journal
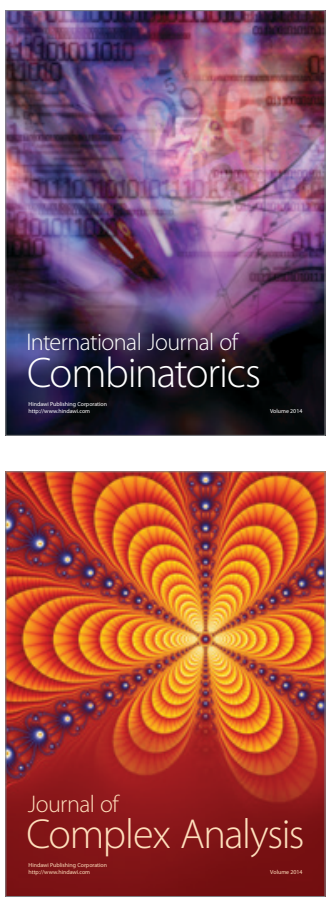

International Journal of

Mathematics and

Mathematical

Sciences
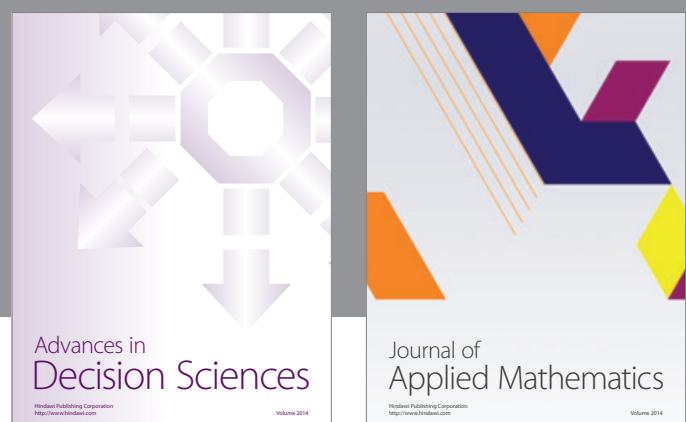

Journal of

Applied Mathematics
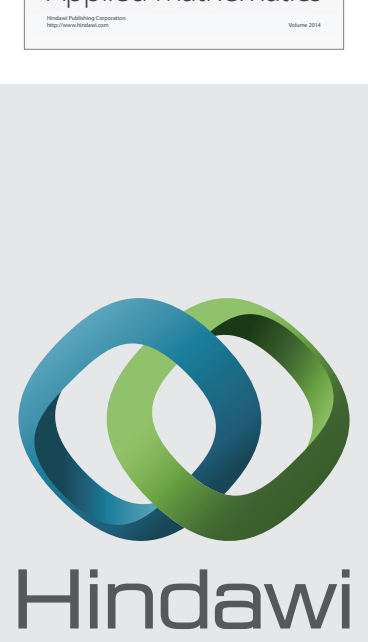

Submit your manuscripts at http://www.hindawi.com
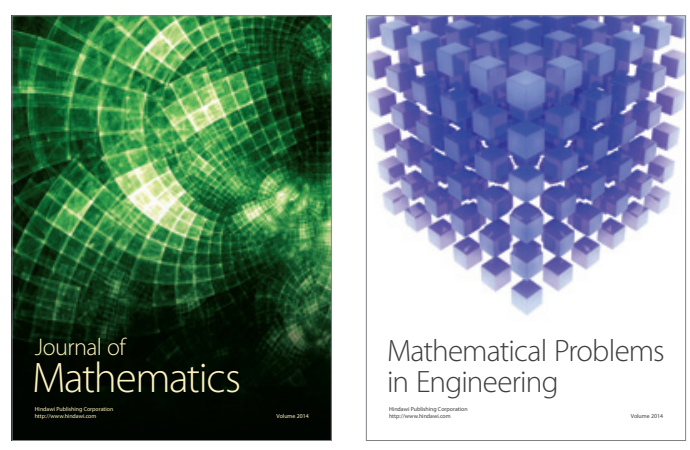

Mathematical Problems in Engineering
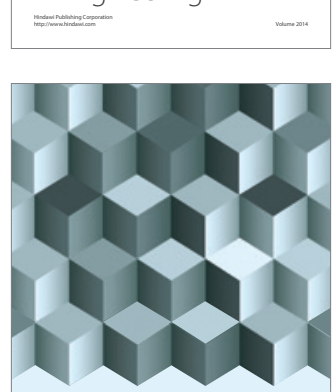

Journal of

Function Spaces
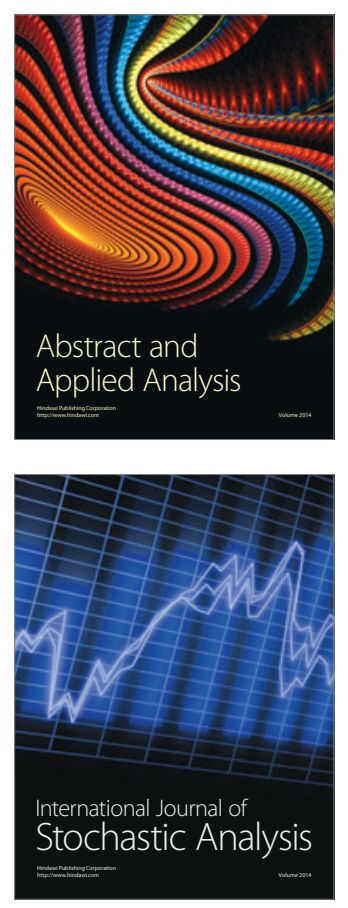

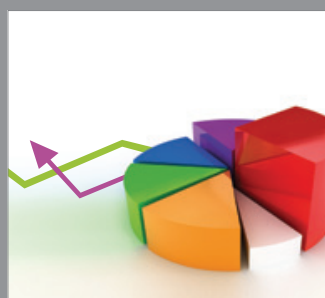

ournal of

Probability and Statistics

Promensencen
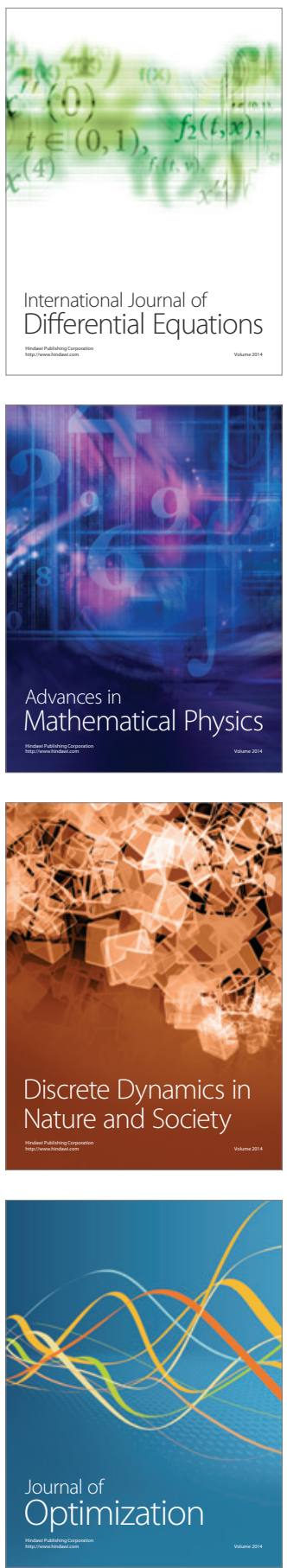